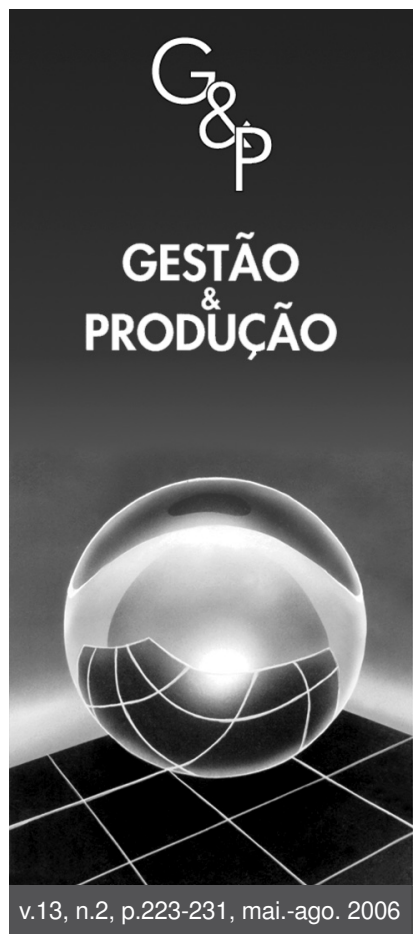

\title{
CONTROLE DE ESTOQUE DE MATERIAIS COM DIFERENTES PADRÕES DE DEMANDA: ESTUDO DE CASO EM UMA INDÚSTRIA QUÍMICA
}

\author{
Antônio Marcos dos Santos \\ lana Araújo Rodrigues
}

Departamento de Engenharia de Produção, Pavilhão Central de Aulas - PCA, Campus da Pampulha, Universidade Federal de Minas Gerais - UFMG, Av. Presidente Antônio Carlos, 6627, CEP 30161-010, Belo Horizonte, MG, e-mails: antoniomimneiro@yahoo.com.br, iana.araujo@gmail.com

\section{Resumo}

O desafio do gestor de estoques é saber quando e quanto ressuprir de cada material e quanto deve manter em estoque de segurança. Com o crescente número de itens com diferentes padrões de demanda e características específicas, a complexidade na administração de materiais aumenta devido à necessidade de controle diferenciado. Este trabalho, por meio de um estudo de caso em uma empresa química, propõe um método de classificação dos materiais em famílias afins com a adoção de políticas distintas de ressuprimento e estoques de segurança, com o objetivo de garantir o balanceamento dos estoques e atender aos níveis de serviço requeridos à produção.

Palavras-chave: classificação multiitens, padrões de demanda, políticas de ressuprimento, estoques de segurança.

\section{Introdução}

A utilização de estoques, seja de segurança ou de cobertura para atender à demanda média durante o lead time é extremamente importante porque possibilita um melhor nível de atendimento ao cliente e melhora a competitividade da empresa em relação aos concorrentes.

Segundo Lenard e Roy (1995), o controle de estoque é estudado desde 1913 com Harris ao introduzir a fórmula do lote econômico de compra. Para uma organização, a otimização do fluxo de materiais é de vital importância, pois os estoques representam grande parte dos seus custos logísticos. Além disso, a produção tem um ritmo que não deve ser interrompido e o custo de manutenção dos estoques representa capital parado que poderia estar sendo utilizado para outros fins. Krever et al. (2003) mostraram em seu estudo que um gerenciamento eficiente de estoques balanceia a disponibilidade de produto, o nível de serviço e os custos de manutenção.

Este trabalho busca desenvolver um sistema de apoio à tomada de decisão para auxiliar no gerenciamento dos estoques de materiais com diferentes padrões de demanda, classificando-os em famílias afins para a aplicação de diferentes políticas de reposição e determinação dos estoques de segurança. $\mathrm{O}$ que se pretende testar neste estudo de caso é se a segmentação dos itens em famílias para controle e reposição vai, efetivamente, reduzir os níveis de estoques de forma balanceada e garantir os níveis de serviço requeridos à produção, estabelecidos como sendo a probabilidade de não faltar material na produção.

A finalidade é relatar a solução de um problema prático de controle de estoques pela aplicação de técnicas combinadas, objetivando a otimização dos estoques da empresa estudada, que é de médio porte e possui uma extensa variedade de matérias-primas e produtos acabados. Como os produtos são para pronta-entrega, de alta substituição e os espaços de armazenagem são limitados, é fundamental um controle eficiente dos estoques de matérias-primas para que não se interrompa o fluxo normal da produção. A falta de matéria-prima altera todo o pla- 
nejamento da produção, gerando mais setups e descontrole na coordenação da capacidade produtiva, além do fato de levar os produtos acabados à ruptura.

A estrutura deste artigo se constitui da seguinte forma: a seção 2 corresponde à revisão da literatura dos assuntos abordados neste trabalho; a seção 3 descreve o estudo de caso; a seção 4 mostra a análise dos resultados; e a seção 5 apresenta as conclusões.

\section{Revisão da literatura}

\subsection{Classificação de estoque multiitens}

Segundo Partovi e Anandarajan (2002), em ambientes com centenas de itens de estoque para controle, o gerenciamento se torna mais complexo devido à diversidade. Uma alternativa é a separação dos itens em subgrupos, permitindo a escolha e adoção da política mais adequada para cada um deles.

A Curva ABC é uma forma de classificação muito utilizada que usa o critério valor de uso anual (quantidade utilizada por ano x valor unitário). Entretanto, essa técnica só é considerada eficiente para classificação de itens quase homogêneos, em que o valor de uso é a principal diferença (Ramanathan, 2004). Por meio da Curva ABC, pode-se dedicar mais atenção aos itens A por representarem alta participação nos valores movimentados de estoque.

Uma grande variedade de itens no estoque aumenta consideravelmente a complexidade do gerenciamento, criando a necessidade de classificá-los com multicritérios. Esses critérios podem ser vários, tais como: lead time, existência de atributos comuns, obsolescência, facilidade de substituição, escassez, durabilidade, distribuição de demanda, dentre outros.

Outros autores abordam a classificação multicritério, dentre eles Hautaniemi e Pirttilä (1999), Guvenir e Erel (1998), Cohen e Ernst (1988) e Huiskonem et al. (2003). Esses autores citam a ferramenta Processo Hierárquico Analítico (AHP) como uma alternativa para a classificação dos materiais. Partovi e Anandarajan (2002) apontam como vantagem da sua adoção o fato de poder incorporar critérios qualitativos e quantitativos e ser fácil de usar, mas o fator emocional pode distorcer os resultados devido à subjetividade dos aspectos qualitativos.

Segundo Lenard e Roy (1995), a maioria dos modelos publicados se concentra em controle de estoque monoitem, mas, na prática, os gestores lidam com centenas de itens. Portanto, defendem um modelo multiitem por 3 razões: i) a agregação dos itens em grupos permite uma economia de tempo que pode ser canalizado para o tratamento dos itens mais importantes; ii) mesmo que os modelos tradicionais proponham soluções para cada item, uma análise ainda deverá ser feita e, no caso de muitos itens, essa análise se torna demorada para ser feita indi- vidualmente; e iii) itens utilizados em grupos funcionais são afetados, simultaneamente, pelas mesmas restrições. Conseqüentemente, os materiais devem ser tratados em famílias distintas, com políticas específicas para a tomada de decisão.

\subsection{A influência dos diferentes padrões de demanda dos produtos na definição das políticas de estoque}

Mudanças no mercado, como crescimento do nível de customização e as fusões e aquisições no mercado industrial, geraram uma demanda extremamente irregular, denominada "lumpy", que é caracterizada pelo alto nível de variabilidade com picos de demanda seguidos de vários períodos de demanda baixa ou nula. Uma forma de medir essa variabilidade da demanda (lumpiness) é usar o coeficiente de variação $(\mathrm{CV})$. A definição do valor de um CV alto, médio ou baixo varia entre os autores (Jacobs e Whybark, 1992). Segundo Pang et al. (2005), o CV é o percentual de variação de uma amostra em relação à sua média, ou seja, é o desvio padrão dividido pela média.

Bartezzghi et al. (1999) comprovaram que as diferentes formas das distribuições da demanda, originadas por essa variabilidade impactam diretamente os níveis dos estoques. Daí, a definição da política de ressuprimento ser um problema desafiador, pois os estoques geralmente operam em presença de eventos aleatórios, tais como: falta de evidência, falta de certeza das evidências e sob informações imprecisas (Petrovic e Petrovic, 2001).

De acordo com Yeh et al. (1997), para se determinar a probabilidade de ruptura do estoque, é preciso descobrir qual distribuição de probabilidade rege a amostragem. Ainda, a distribuição Gamma é obtida quando períodos com demandas nulas e com intervalos entre demandas também pequenos têm maior freqüência de incidência.

Hautaniemi e Pirttilä (1999) fazem uma comparação entre políticas de controle de estoques tais como MRP, Ponto de Ressuprimento (PR) e sistemas de revisões visuais como "two-bin" e dizem ser, equivocadamente, consideradas mutuamente excludentes pela literatura.

\subsection{Estoque de segurança e ponto de ressuprimento}

Os estoques de segurança existem por causa das incertezas da demanda e do lead time de fornecimento. Segundo Ballou (2001), se a demanda fosse determinística e a reposição fosse instantânea, não haveria a necessidade desse tipo de estoque.

De acordo com Buzzacott e Shanthikumar (1994), uma alternativa para reduzir estas incertezas é o tempo de segurança que é aplicável quando se tem uma previsão bem acurada, variando apenas o lead time. Entretanto, flutuações da demanda durante o lead time diminuem sua atratividade por considerar a demanda constante. $\mathrm{O}$ 
tempo de segurança, que é o desvio padrão do lead time $\left(E S=\sigma_{l t}\right)$, incrementa o lead time de entrega médio, da mesma forma que o estoque de segurança incrementa a demanda média durante o lead time. Quando a demanda de um material segue uma distribuição Normal, o cálculo do estoque de segurança pode ser feito pelo desvio padrão da soma das variâncias do lead time e da demanda:

$$
E S=\left\{\sqrt{\left(\sigma_{d}\right)^{2} L T+\left(\sigma_{L T}\right)^{2} d^{2}}\right\} * Z
$$

\section{Em que,}

LT é o tempo de reabastecimento; $\left(\sigma_{\mathrm{d}}\right)^{2}$ é variância da demanda durante o lead time; $\left(\sigma_{\mathrm{LT}}\right)^{2}$ é a variância do lead time de entrega dos fornecedores; e $\mathrm{Z}$ é o número de desvios tabelado que garante o nível de serviço requerido.

Krupp (1997) propõe uma forma de cálculo, considerando o $\mathrm{Z}$ como o ponto de equilíbrio entre o valor investido em estoque de segurança e o lucro recuperado por vendas que seriam perdidas.

Segundo Inderfurth e Minner (1998), esta fórmula é aplicada quando os comportamentos da demanda e do lead time obedecem a uma distribuição Normal. Na prática, essa normalidade da distribuição não é garantida, o que leva a rupturas no estoque (Eppen e Martin, 1988).

Eppen e Martin (1988) complementam dizendo que somente em alguns casos se pode encontrar um material com uma distribuição Normal de demanda. Dada essa dificuldade, o procedimento padrão é assumir a distribuição como sendo Normal com média e variância conhecidas para determinar o estoque de segurança e o ponto de ressuprimento. Este procedimento facilita, mas infelizmente incorre em erros, levando a rupturas no estoque ou a excesso do mesmo.

Ainda, segundo Krupp (1997), quando as distribuições são desconhecidas, o que é comum, e algum método de estimativa é usado para calcular a demanda em cada período, o sistema pode computar os erros médios absolutos da previsão (MAD) em relação à demanda real e usá-los para definir os estoques de segurança, calculado da seguinte maneira:

$$
E S=k *(M A D) * \sqrt{L t}
$$

Sendo que,

$$
M A D=\frac{\sum_{i=1}^{n}\left|x_{i}-u_{i}\right|}{n}
$$

Em que,

$u_{\mathrm{i}}$ é a previsão para o período $\mathrm{i}$; $x_{\mathrm{i}}$ é a demanda real no período i; n é o total de períodos considerados; e k é o coeficiente de proporcionalidade do estoque de segurança, sendo encontrado numa tabela específica apresentada por Krupp (1997).
Chan et al. (1999) afirmam que, em muitas empresas, os estoques de segurança estão diretamente relacionados ao desvio padrão da previsão e que há vários estudos que combinam diferentes modelos de previsão com obtenção de melhores resultados.

Segundo Inderfurth e Minner (1998), usualmente, a proteção contra as incertezas não é conseguida somente com os estoques de segurança, mas pelo esforço gerencial, da flexibilidade e da capacidade de resposta dos processos para reagir a uma situação inesperada.

Para a definição do ponto de ressuprimento, o estoque de segurança deve ser somado com a demanda durante o lead time, independente da fórmula de cálculo (Ballou, 2001).

Krever et al. (2003) propõem uma expressão analítica para o ponto de ressuprimento baseados nas demandas individuais com comportamento esporádico. Haksever e Moussourakis (2003) apresentam um modelo para lidar com o problema do ressuprimento de materiais sujeito a algumas restrições de tempo, espaço e recursos financeiros.

\subsection{Outros aspectos ligados ao gerencia- mento de estoques}

Lenard e Roy (1995) apresentam algumas críticas referentes aos modelos clássicos de estoque: i) os custos de pedido, de manutenção e de falta são difíceis de serem mensurados; ii) a complexidade para serem compreendidos requer um relaxamento na implementação, o que enfraquece o realismo dos modelos; e iii) são inflexíveis e fazem uma confusão entre variáveis de ação (níveis de ressuprimento e lotes de compra) e de monitoramento (níveis de estoque, nível de serviço ao cliente e carga de trabalho) no controle de estoques. Portanto, para os autores, existe uma grande lacuna entre teoria e prática.

Para Zomerdijk e Vries (2003), esses modelos e técnicas normalmente são baseados em modelagem matemática e são bons na determinação de parâmetros de estoques e planejamento de recursos, porém, quando os modelos são confrontados com o mundo real, apresentam grandes limitações.

Atualmente, é reconhecido pelas empresas que o desempenho dos sistemas de estoque não depende somente de como são planejados ou controlados, mas também da arquitetura organizacional. Por isso, muitas delas, com o intuito de melhorá-lo, implementam, simultaneamente, avançados sistemas com o uso de medidas organizacionais (Vries, 2004).

\section{Estudo de caso}

O estudo de caso foi realizado numa empresa de médio porte que fabrica e comercializa produtos químicos para limpeza doméstica e industrial. As matérias-primas totalizam 508 itens com as mais diversas características. 
Grande parte dos custos da empresa está ligada à manutenção dos estoques para o atendimento da demanda. $\mathrm{O}$ histórico da empresa é de alto índice de avarias e grandes divergências nos inventários, causados pelo descontrole e grandes estoques, gerando falta de espaço para organização e movimentação. Ainda, há a perturbação da produção por falta de material, enquanto há materiais se deteriorando pelo tempo de estocagem.

Para solucionar esses problemas, os itens foram classificados em famílias por similaridade, definindo as políticas de ressuprimento e estoques de segurança de acordo com os níveis de serviço requeridos. Esse procedimento busca otimizar os estoques, eliminando os excessos e garantindo os níveis de serviços. A Figura 1 apresenta a classificação geral.

Em que, LT é o lead time de entrega; HP é o horizonte de planejamento; IEC é o intervalo entre consumos.

Inicialmente, foi feita uma classificação dos materiais entre comuns e especiais de acordo com a experiência dos gestores. O critério de separação foi considerar como especiais aqueles itens com características distintas que os fazem destoar essencialmente dos demais. Os comuns se diferenciam apenas pelo padrão de demanda, importância e valor movimentado por período.

Posteriormente, foi feita uma classificação $\mathrm{ABC}$ dos materiais de acordo com o seu valor mensal movimentado. Os itens A e B foram tratados separadamente dos $\mathrm{C}$, para canalizar atenção aos primeiros, devido ao alto valor financeiro que representam, além de serem vitais para o funcionamento da empresa. Apenas $12 \%$ dos 508 itens representaram $80 \%$ do valor anual movimentado (itens A). Em contrapartida, outros $66 \%$ dos itens representou apenas 5\% desse valor movimentado (itens C). Daí a importância de se tratar os itens A com mais atenção, pois qualquer otimização no seu controle terá grande impacto no estoque médio, medido em Reais.

Para os itens A e B, foi feito um levantamento de dados de demanda semanal dos materiais de jan/2001 a jul/2005. Esses foram extraídos do banco de dados da empresa e submetidos ao software Best Fit versão 2.0d para análise da distribuição da demanda, que foi utilizada como outro critério hierárquico para estratificação dos itens após a classificação ABC. Esse software ajusta os dados coletados às curvas e faz os testes de aderência (neste trabalho, o teste utilizado foi o Qui-quadrado). A Figura 1 identifica as distribuições encontradas (Beta, Gamma e Normal) para alguns itens e, também, aqueles itens que não se ajustaram a nenhuma distribuição e foram considerados como irregulares.

Dos 508 itens, 28 são fabricação própria; 5 novos, 1 perecível, 1 abastecimento contínuo, 2 sazonais; 328 são C não-vitais, 7 são C vitais, 6 têm LT > HP, 32 são Normais, 19 são Beta/Gamma com LT < IEC, 10 são Beta/Gamma com LT > IEC, 48 são irregulares com LT < IEC e 21 são irregulares com LT $>$ IEC.

Uma filtragem dos dados foi feita para eliminar todas as discrepâncias não explicadas e que afetam as análises. As distribuições dos lead times foram consideradas normais porque os fornecedores tendem a respeitar os prazos de entrega médios previstos nos contratos.

As seções seguintes descrevem os critérios de classificação e as políticas de ressuprimento detalhadas para cada família.

\subsection{Itens especiais}

Conforme a Figura 1, a primeira separação dos materiais em famílias foi entre os itens comuns e os especiais, como sugerido por Hautaniemi e Pirttilä (1999). Os últimos se referem àqueles com características peculiares e que devem ser tratados individualmente, tais como fabricação própria, itens novos, perecíveis, abastecimento contínuo e sazonal. Essas famílias foram definidas de acordo com a experiência dos gestores.

Os itens de fabricação própria são produzidos de acordo com a necessidade de ressuprimento estabelecida pelo departamento de Planejamento e Controle da Produção

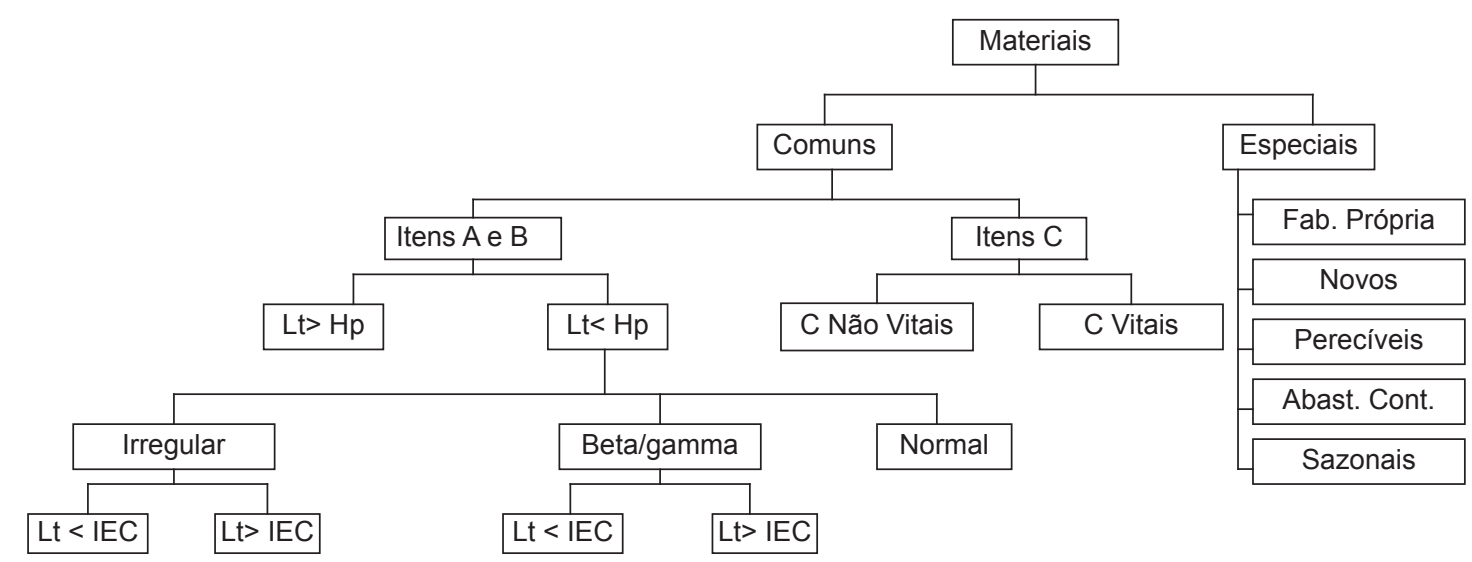

Figura 1. Classificação geral dos materiais. 
(PCP). Esses itens são ressupridos sob pedido, de acordo com a política MTO (Make to Order), nas quantidades exatas necessárias.

Os itens novos não têm história e a única referência que o gestor de materiais tem para efetuar as primeiras compras é a previsão de vendas, baseada em pesquisas de mercado, fornecida pelo departamento de Marketing. Normalmente, a partir do terceiro mês, a demanda do item começa a se estabilizar e deixa de ser especial. A compra desses materiais é acrescida de uma margem de segurança definida pelo gestor.

Os itens perecíveis não podem ser mantidos em estoque, sendo requisitados no momento em que serão consumidos. A política de ressuprimento é, pois, MTO, já que o ressuprimento só é feito quando uma requisição firme da produção é realizada. Chang et al. (2003) enfatizam que essa política prioriza a data programada, ou seja, a previsão tem que ser bem acurada.

A água está presente na composição de $97 \%$ dos produtos e tem abastecimento contínuo por meio da companhia de abastecimento da cidade, o que dispensa quaisquer políticas de ressuprimento.

A última família de itens especiais é formada por aqueles que possuem fornecimento sazonal, ou seja, a disponibilidade de fornecimento é limitada a alguns períodos do ano quando ocorre a safra. A política adotada é a revisão periódica (REV), já que a compra é feita anualmente. A quantidade comprada é obtida calculando o consumo médio até a próxima safra com uma margem de segurança, proveniente do erro da previsão (MAD).

\subsection{Itens comuns}

Os itens comuns são aqueles que não apresentam nenhuma característica especial que os distinga dos demais. O que os diferencia entre si são os padrões de demanda. Existem os chamados fast moving e os slow moving. Os primeiros são aqueles de alto giro e padrões de demanda regulares, tendendo para o contínuo. Os outros são os de baixo giro e demanda irregular, ou seja, existem vários períodos em que não há registro de seu consumo. Dentro dessa classificação, primeiramente, houve a separação dos itens entre A-B e C.

\subsubsection{Itens $C$}

Os itens $\mathrm{C}$ foram subdivididos em $\mathrm{C}$ vitais e $\mathrm{C}$ não vitais, tendo como critério de separação a regularidade do consumo. Os $\mathrm{C}$ vitais se ajustam à distribuição Normal, pois têm demanda contínua com períodos de demanda nula desprezíveis. Já os $\mathrm{C}$ não vitais não se ajustam à distribuição Normal, pois possuem períodos consideráveis de demanda nula e irregular.

Os itens $\mathrm{C}$ vitais, apesar do baixo valor movimentado, são críticos para o funcionamento da empresa e sua falta acarreta parada generalizada da produção. Esses itens têm consumo contínuo, são utilizados em vários produtos acabados e não têm substitutos. São baratos e importantes, por isso, a adoção de estoques de segurança maiores, calculados pela fórmula tradicional usada para a distribuição Normal com nível de serviço de $99 \%$, vai prover segurança sem onerar os estoques. A política de ressuprimento adotada foi Ponto de Ressuprimento (PR).

Os Itens $\mathrm{C}$ não vitais têm demanda irregular e sua importância unitária no conjunto é pequena. Normalmente, são consumidos em poucos produtos acabados e sua falta é absorvida pela flexibilidade da produção. A política de ressuprimento escolhida também foi PR. Essa política é normalmente aplicada quando o material tem uma demanda regular, caso contrário, tem sua eficiência reduzida. Mesmo assim, foi adotada como forma de otimização do tempo do gestor para permitir-lhe se ocupar com os outros itens mais relevantes, conforme sugerido por Hautaniemi e Pirttilä (1999). Os estoques de segurança foram medidos pelo erro médio da previsão em relação ao realizado (MAD). Como são itens baratos e não tão importantes, o nível de serviço adotado foi de $95 \%$.

\subsubsection{Itens A e B}

Os itens A e B foram divididos comparando o lead time de fornecimento (LT) de cada material com o horizonte de planejamento da produção (HP). O HP da empresa é de 30 dias. Logo, qualquer política de ressuprimento que dependa da previsão da produção ou consumo do item para mais de 30 dias não pode ser aplicada. Nesse caso, materiais com LT > HP, a política adotada foi a revisão periódica que se baseia no histórico para definição de parâmetros de controle. Seu estoque de segurança foi calculado pelo erro médio da previsão de consumo em relação ao realizado (MAD). Produtos com grandes lead times normalmente apresentam maior incerteza na entrega, logo, adotou-se um nível de serviço de $98 \%$.

Os itens A e B com LT < HP foram subdivididos de acordo com o ajuste às distribuições de probabilidade realizado com a utilização do software Best Fit. As distribuições encontradas foram Normal, Beta e Gamma. Os itens cujas demandas não se ajustaram a nenhuma distribuição foram denominados irregulares. Geralmente, os itens fast moving foram aqueles de consumo contínuo regular regidos pela distribuição Normal, enquanto os itens slow moving foram aqueles de consumo intermitente e esporádico e que não se ajustaram a nenhuma distribuição. Entre estas duas faixas, existem aqueles itens com padrão de consumo intermitente, entretanto, sistemático e regular que se ajustaram às distribuições Beta e Gamma. A Figura 2 representa graficamente os padrões de demanda encontrados, distintos por sua continuidade e regularidade.

Em que o item: i) Contínuo, é consumido toda semana; ii) intermitente, não é consumido toda semana; 
iii) regular, tem consumo sistemático (ciclo constante); e iv) irregular, tem consumo esporádico (ciclo variável).

A associação dos padrões de demanda com a curva $\mathrm{ABC}$, tal qual mostrado na Figura 2, se constituiu como forte tendência no estudo de caso, entretanto, pode ocorrer em alguns casos de um material estar na classe $\mathrm{C}$ e possuir um padrão de demanda contínuo e regular.

À família dos itens com distribuição Normal foi aplicada a política PR, com nível de serviço de $95 \%$ e o estoque de segurança calculado pela fórmula tradicional. Como a demanda é contínua, o intervalo médio entre consumos (IEC), nesse caso, se torna irrelevante.

Os itens com distribuição de demanda Beta ou Gamma, apesar de se ajustarem a essas distribuições contínuas, podem ter vários períodos com demanda nula. Para tratá-los então, foi instituída uma regra: se o LT < IEC, adota-se a política Planejamento para trás (PPT) para ressuprimento. Caso contrário, adota-se PR. O PPT utilizarse-á da previsão de consumo de cada item oriunda do planejamento da produção. Por meio dessas datas, faz-se um planejamento para trás para se determinar a hora de colocar o pedido de reposição do material.

Para esses itens, foi adotado o tempo de segurança, pois como a previsão de demanda é muito acurada, ou seja, o PCP tem boa precisão quanto ao seu planejamento, o erro da previsão é desprezível e está dentro da margem de flexibilidade do planejamento de produção. Com isso, ocorre apenas o desvio padrão do lead time, já que a única incerteza que resta é a variação do tempo de entrega. Já o restante, $\mathrm{LT}>$ IEC, o estoque de segurança foi calculado pelo MAD. Ambos têm um nível de serviço de $95 \%$.

Aos itens com padrões de demanda irregulares foi aplicada a mesma regra dos materiais com distribuição de demanda Beta ou Gamma.
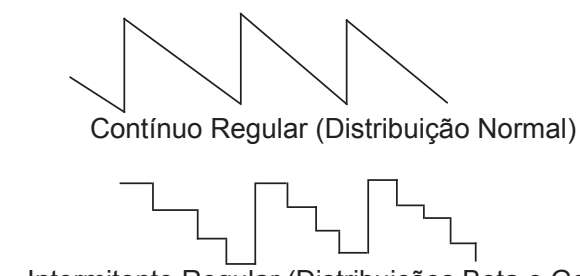

Intermitente Regular (Distribuições Beta e Gamma)

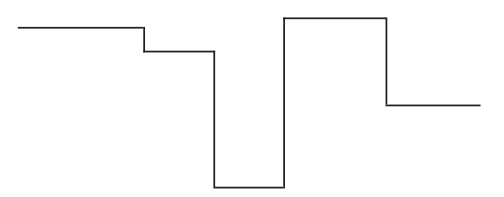

Intermitente Irregular (Não se ajustaram) Itens "slow moving"

Figura 2. Padrões de demanda típicos encontrados.

\section{Análise dos resultados}

Para verificar a aderência do sistema de apoio à tomada de decisão desenvolvido com a realidade, foi feita uma comparação da evolução dos níveis de estoque médios de acordo com determinados níveis de serviço antes e após a implementação do sistema (Figura 3). Vale ressaltar que foram mantidos os mesmos lotes de compra já praticados pela empresa, com o intuito de não influenciar nos resultados. O impacto dos níveis de serviço alcançados é medido pelo número de horas produto paradas (HPP). Esse cálculo leva em consideração a quantidade de produtos que utilizam cada item. Por exemplo: se um material faltar por 5 horas e for usado em 3 diferentes produtos acabados, sua falta acarretará, então, 15 horas produto paradas.

No estudo de caso realizado, foram obtidas 13 famílias diferentes de acordo com o método de classificação estabelecido. Para cada família, foram calculados o nível de segurança e a política de ressuprimento. Foram adotadas quatro diferentes políticas de ressuprimento: Ponto de Ressuprimento (PR); Planejamento para Trás (PPT); Make to Order (MTO); e Revisão Periódica (REV). As três formas para cálculo do estoque de segurança foram:
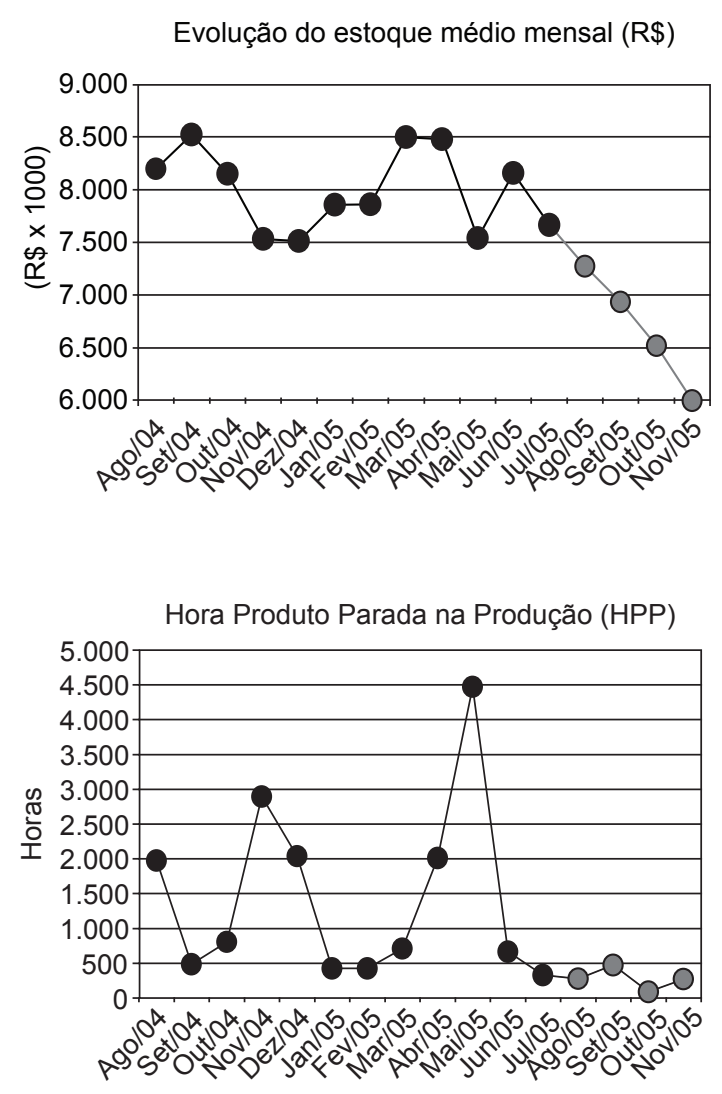

Figura 3. Comparativo do estoque médio e HPP antes e depois da implementação do sistema de controle. 
Tempo de segurança, MAD e Estoque de segurança clássico para a distribuição Normal.

Para os itens de consumo regular que se ajustaram à distribuição Normal, foi aplicada a fórmula tradicional do Estoque de Segurança. Para aqueles que não se ajustaram, se o lead time fosse menor que o ciclo de consumo (IEC), foi adotado o tempo de segurança. Para os demais, adotou-se o erro médio absoluto (MAD) da previsão mensal, por não se ter uma regularidade da demanda tal quais os outros materiais.

Este trabalho tem duas ênfases básicas: definição das políticas de ressuprimento e cálculo dos estoques de segurança. O objetivo fundamental dessas duas abordagens é reduzir o estoque médio com a definição do tempo ótimo, para colocar as requisições e o dimensionamento preciso dos estoques de segurança de acordo com os níveis de serviço requeridos. Portanto, ao se tratar os itens de maneira específica, levando em consideração os diferentes padrões de demanda, o sistema é otimizado como um todo.

Na Figura 3, dados fornecidos pelo sistema desenvolvido, é possível observar que houve uma redução gradativa do estoque médio da empresa desde a implantação do sistema (ago/05), sendo que as horas produto paradas (HPP) na produção permaneceram praticamente inalteradas dentro de níveis perfeitamente aceitáveis pelo gestor de PCP (abaixo de 5\%).

Segundo o gestor de produção, quando o nível de serviço atinge $95 \%$ (em torno de $500 \mathrm{HPP}$ ), a perturbação pela falta de material na produção é perfeitamente coberta pela flexibilidade do planejamento. Diante disso, todos os níveis de serviço para o cálculo dos estoques de segurança propostos pelo gestor de produção neste estudo de caso são, no mínimo, $95 \%$.

A determinação das diferentes políticas de estoque, por meio do estudo do comportamento da demanda, permitiu um balanceamento dos estoques ao mesmo tempo em que possibilitou atender ao nível de serviço mínimo de $95 \%$ requerido pela produção. Esse balanceamento é fundamental para a estabilidade do processo produtivo. Além disso, possibilita ganho de espaço físico e otimiza os investimentos em estoque.

Para ficar mais interativo com o usuário, o sistema de controle de estoques dispõe de sinalizadores visuais (Figura 4) que indicam a situação dos estoques e das requisições de cada item, alertando o gestor de materiais sobre: i) a hora de repor os estoques; ii) as requisições atrasadas ainda não realizadas; iii) as requisições feitas com atraso; e iv) o item cujo estoque atingiu o nível de segurança e pode entrar em ruptura. Além disso, o sistema dispara automaticamente um e-mail para o gestor de materiais quando um item atinge seu ponto de ressuprimento.

Os parâmetros estoque de segurança e ponto de ressuprimento são atualizados automaticamente, permitin-
(i) $\oplus$ Requisitar hoje
(ii) Requisição em atraso ainda não feita

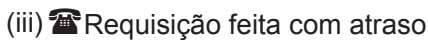
(iv) $£$ Estoque crítico

Figura 4. Sinalizadores visuais para controle de reposição de materiais.

do que o gestor otimize seu tempo, atendo-se apenas à análise crítica das soluções apontadas e se aplique nas questões gerenciais estratégicas. Entretanto, devido ao sistema de manufatura estar vulnerável à ação externa de eventos aleatórios e situações inesperadas, é fundamental o seu acompanhamento constante para que sejam feitas, antecipadamente, todas as correções de planejamento, como forma de diminuição das incertezas que geram estoques.

\section{Conclusões}

O método proposto neste trabalho foi eficaz no balanceamento e redução dos níveis de estoques médios e manutenção dos níveis de serviço à produção. Em 4 meses o estoque médio da empresa foi reduzido em $34 \%$, passando de $\mathrm{R} \$ 7.600 .000,00$ para cerca de $\mathrm{R} \$ 6.000 .000,00$ e a quantidade de Horas Produto Paradas na produção permaneceu constante dentro de níveis aceitáveis pela empresa. Os reflexos práticos foram imediatos: além da disponibilização de capital para outros fins, o arranjo físico dos materiais foi beneficiado, diminuindo as avarias por acúmulo; os inventários periódicos se tornaram mais fáceis por estarem mais organizados, diminuindo drasticamente as divergências.

Outro aspecto importante que merece ser destacado é que todos os processos da empresa agora precisam estar mais integrados, pois as deficiências que os altos níveis de estoques encobriam, agora, começam a aparecer e precisam ser resolvidas em vários departamentos. A empresa ganhou maior eficiência operacional com este conceito de redução de desperdício e melhoria dos processos, justamente o que prega a teoria do Just in Time tão difundida nas empresas atualmente.

Apesar dos resultados apresentados, a gestão do estoque ainda necessita de acompanhamento contínuo, de atualização de parâmetros, de antecipação aos problemas e de entrosamento, pois o ambiente não é estacionário. Os padrões de demanda variam com o tempo, novos itens são introduzidos e alguns substituídos. Além disso, eventos inesperados acontecem freqüentemente no chãode-fábrica o que altera o planejamento de reposição de materiais.

A maior dificuldade encontrada foi a triagem dos dados e estabelecimento dos critérios para classificação dos 
itens. O bom senso ajuda muito, considerando que cada empresa é um caso diferente.

A adoção da política de ressuprimento adequada para cada material com diferente padrão de demanda e de estoque de segurança permite uma otimização do estoque e garante os níveis de serviço requeridos. A utilização de uma boa ferramenta de suporte à tomada de decisão, ali- nhada com os objetivos da organização, é um fator que contribui muito para a competitividade. É importante que não só a área de materiais seja otimizada, mas a cadeia de suprimentos como um todo, desde o desenvolvimento de fornecedores, planejamento da produção até a distribuição de produtos aos clientes, o que é assunto para trabalhos futuros.
BALLOU, R. H. Gerenciamento da cadeia de suprimentos. Planejamento, organização e logística empresarial. 4. ed. São Paulo: Bookman, 2001.

BARTEZZGHI, E.; VERGANTI, R.; ZOTTERI, G. Measuring the impact of asymmetric demand distributions on inventories. International Journal of Production Economics, v. 60-61, p. 395-404, 1999.

BUZZACOTT, J. A.; SHANTHIKUMAR, J. G. Safety stock versus safety time in MRP controlled production systems. Management Science, v. 40, n. 3, p. 1678-1689, 1994.

CHAN, C. K.; KINGSMAN, B. G.; WONG, H. The value of combining forecasts in inventory management - a case study in banking. European Journal of Operational Research, v. 117, p. 199-210, 1999.

CHANG, S. U.; PAI, P. F.; YUAN, K. J.; WANG, B. C.; LI, R. K. Heuristic PAC model for hybrid MTO and MTS production environment. International Journal of Production Economics, v. 85, p. 347-358, 2003.

COHEN, M. A.; ERNST, R. Multi-item classification and generic inventory stock control policies. Production and Inventory Management Journal, v. 29, n. 3, p. 6-8, 1988.

EPPEN, G. D.; MARTIN, R. K. Determining safety stock in the presence of stochastic lead time and demand. Management Science, v. 34, n. 11, p. 1380-1390, 1988.

GUVENIR, H. A.; EREL, E. Multicriteria inventory classification using a genetic algorithm. European Journal of Operational Research, v. 105, p. 29-37, 1998.

HAKSEVER, C.; MOUSSOURAKIS, J. A model for optimizing multi-product inventory systems with multiple constraints. International Journal of Production Economics, v. 97, p. 18-30, 2003.

HAUTANIEMI, P.; PIRTTILÄ, T. The choice of replenishment policies in an MRP environment. International Journal of Production Economics, v. 59, p. 85-92, 1999.

HUISKONEM, J.; NIEMI, P.; PIRTTILÄ, T. An approach to link customer characteristics to inventory decision making. International Journal of Production Economics, v. 81-82, p. 255-264, 2003.

INDERFURTH, K.; MINNER, S. Safety Stocks in multistage inventory systems under different service measures. European Journal of Operational Research, v. 106, p. 57-73, 1998.

JACOBS, F. R.; WHYBARK, D. C. A Comparison or Reorder Point and Material Requirements Planning Inventory Control Logic. Decision Sciences, v. 23, n. 2, p. 332-342, 1992.

KREVER, M.; WUNDERINK, S.; DEKKER, R.; SCHORR, B. Inventory control based on advanced probability theory, an application. European Journal of Operational Research, v. 162, n. 2, p. 342-358, 2003.

KRUPP, J. A. G. Safety stock management. Production and Inventory Management Journal, v. 38, n. 3, p. 11-18, 1997.

LENARD, J. D.; ROY, B. Multi-item inventory control: A multicriteria view. European Journal of Operational Research, v. 87, p. 685-692, 1995.

PANG, W. K.; LEUNG, P. K.; HUANG, W. K.; LIU, W. On interval estimation of the coefficient of variation for the three-parameter Weibull, Lognormal and Gamma distribution: A simulation-based approach. European Journal of Operational Research, v. 164, n. 2, p. 367-377, 2004.

PARTOVI, F. Y.; ANANDARAJAN, M. Classifying inventory using an artificial neural network approach. Computers \& Industrial Engineering, v. 41, p. 389-404, 2002.

PETROVIC, R.; PETROVIC, D. Multicriteria ranking of inventory replenishment policies in the presence of uncertainty in customer demand. International Journal of Production Economics, v. 71, p. 439-446, 2001.

RAMANATHAN, R. ABC inventory classification with multiple-criteria using weighted linear optimization. Computers \& Operations Research, v. 33, n. 3, p. 695-700, 2004. 
VRIES, J. The complex relationship between inventory control and organizational setting: Theory and practice. International Journal of Production Economics, v. 93-94, p. 273-284, 2004.

YEH, Q. J.; CHANG, T. P.; CHANG, H. C. An inventory control model with Gamma distribution. Microelectronics and Reliability, v. 37, n. 8, p. 1197-1201, 1997.
ZOMERDIJK, L. G.; VRIES, J. An organizational perspective on inventory control: Theory and a case study. International Journal of Production Economics, v. 81-82, p. 173-183, 2003.

\section{INVENTORY CONTROL OF ITEMS WITH DIFFERENT DEMAND PATTERNS: A CASE STUDY IN THE CHEMICAL INDUSTRY}

\section{Abstract}

The main challenge of inventory managers is to define when and how many of each item to replenish and how much to keep in a safety stock. The increasing number of items with different demand patterns and specific characteristics requires different policies to ensure a good performance in this area. Based on a case study of a chemical company, this paper proposes a method for classifying materials with different demand patterns into families, using specific replenishment and safety stock policies aimed at maintaining a balance of stocks and meeting the service levels required for production.

Keywords: multi-item classification, demand patterns, replenishment policies, safety stocks. 
